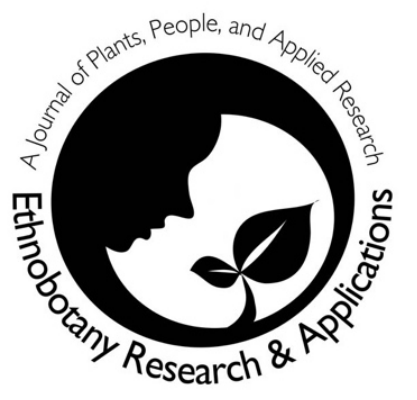

\title{
Emilia Romagna and Malta: A comparative ethnobotanical study
}

\author{
Galuzzo F., Attard E. and Di Gioia D.
}

\section{Correspondence}

Galuzzo F. ${ }^{1}$, Attard E. ${ }^{{ }^{*}}$, and Di Gioia D. ${ }^{1}$

${ }^{1}$ Scuola di Agraria e Medicina Veterinaria, Alma Mater Studiorum-Università di Bologna, Italy

${ }^{2}$ Division of Rural Sciences and Food Systems, Institute of Earth Systems, University of Malta, Malta

*Corresponding Author: everaldo.attard@um.edu.mt

Ethnobotany Research \& Applications 22:14 (2021)

\section{Research}

\begin{abstract}
Background: A comprehensive ethnobotanical study was conducted on two Mediterranean regions which crossed over in the past through several cultures. Although the two regions have distinctive geographical and cultural characteristics, the aim of this study was to determine potential ethnobotanical similarities between the two regions.
\end{abstract}

Methods: This desk research involved the thorough examination of the floral species that thrive in these two regions and ethnobotanical information collected from reliable sources. Once the information was collected, the data was sorted and organized into matrices and then analyzed statistically.

Results: Following a thorough search for common plants within the two regions, 193 taxa, distributed in 72 plant families, were identified. The three predominant families, Asteraceae, Lamiaceae and Apiaceae, were selected according to the popularity of medicinal uses of the taxa within the families $(10.4 \%, 7.8 \%$ and $5.2 \%$, respectively). The popular therapeutic ratios for taxa within the Asteraceae, Lamiaceae and Apiaceae families varied between 0.14-1.00, 0.17-0.82 and 0.27-0.60, respectively. It was observed that the most targeted therapeutic system was the gastrointestinal system covering several ailments that included gastric disturbances, liver and biliary conditions, and intestinal problems.

Conclusions: It can be concluded that although, these two regions are distinct from each other, the two regional communities share the common understanding that the health status of an individual depends on the maintenance of the health of the digestive system. Today, several studies support this relationship, with scientific evidence and the use of natural products for their medicinal, nutraceutical and functionality in everyday life.

Key words: Ethnobotany, Folk medicinal plants, Malta, Italy, Asteraceae, Lamiacea, Apiaceae.

\section{Background}

Several researchers in different parts of the world had delved into the study of the relationship between communities and plants, to understand the importance and potential use of plants in today's world. This multidisciplinary research provides the basis for the discovery of new drugs (Agelet \& Vallès 2003). Research is conducted either on a particular region or locality (Novias et al. 2004; Rivera et al. 2005; Akerreta et al. 2007, Maxia 
et al. 2008, Carrió \& Vallès 2012, Nawash et al. 2014, Bulut et al. 2017) or on several regions or localities (GonzálezTejero et al. 2008, Hadjichambis et al. 2008, Pieroni \& Cattero 2019) to compare and contrast medicinal plant use between different cultures.

Since antiquity the Mediterranean region was considered as a centre of cultural diversity and traditions. Amongst these cultures, prominent ones included the Egyptian civilization, the Phoenicians, the Greeks, the Romans, and the Arabs (Savo et al. 2010, https://www.academia.edu/22703833/Plants_history_and_cultures_in_the_Mediterranean_ area). It was also considered as a centre of trade linking southern Europe, North Africa, and western Asia together. Trade of goods included several plants that have naturalized and formed part of the flora. Knowledge on the use of these plants has been transmitted horizontally between cultures and vertically from one generation to the next within a culture. Migration is another factor that has contributed to spreading of such knowledge. However, the density and abundance of certain taxa varied significantly from region to region and from country to country. Several taxa have evolved into genetically different chemotypes with different edible and therapeutic uses. This ultimately affected the popularity of plants for their use in tradition and instigates further research towards plant genetic resources (Laghetti et al. 2004).

The Mediterranean bioclimate is one of the five bioclimates traditionally recognized in Europe. This climate is defined as 'an extratropical climate with seasonal and daily photoperiodicity, with rainfall concentrated in the cold or relative cold seasons of the year, summer, the hottest season, being dry' (Emberger 1954). Other authors attempted to improve on this definition keeping Emberger's definition as the basis for their description (Di Castro 1981, Rivas-Martinez et al. 2011). Although the Mediterranean bioclimate is also described in several other regions, such as Australia, California, Chile and South Africa, the territory of the Mediterranean Basin is the largest area that represents this type of bioclimate (Sánchez-Mata \& Morales 2016). This region is characterized by four major peninsulas (Italian, Iberian, Balkan, and Anatolian) on the southern European side connected eastwards to the Near East which is connected westwards to the North African region in a clockwise manner. In the Mediterranean region, plants have naturalized in different biomes namely, forests, woodlands, scrubs, and deserts. The diverse topography leads to a rich Mediterranean biome, which is characterized by forests, woodlands, and scrub. Greuter (1991) reported the presence of more than 24,000 floral taxa along the Mediterranean area, hence showing the suitability of the Mediterranean conditions to a vast range of organisms.

The histories of Malta and Italy cross at several points in time. In 218 B.C., Malta was passed on to the Roman Empire at the beginning of the Second Punic War. In 1091 A.D., Malta was invaded by the Normans and was officially part of the Kingdom of Sicily. Another important cross was during the rule of the Order of Saint John, particularly during the Great Siege of 1565 A.D. The Knights made the Italian language as the official language till 1798 A.D. During the British rule from 1800 A.D., political organizations were created, in part, to protect the Italian language in Malta.

The aim of this study is to determine any similarities and differences in the usages of medicinal plants belonging to two different geographical areas in two neighboring countries separated by sea.

\section{Materials and methods}

Study areas

The two geographic regions considered in this study are situated in two different countries, but both belong to the Mediterranean region (Figure 1). The Maltese Islands constitute a land area of $316 \mathrm{~km}^{2}$. With a population of about 515,000 , the population density is currently 1,282 inhabitants per $\mathrm{km}^{2}$ (NSO 2020, https://nso.gov.mt/en/News_Releases/Documents/2020/07/News2020_114.pdf). The highest point is at $253 \mathrm{~m}$ above mean sea level.

With nine provinces within the Emilia-Romagna region (within the Italian peninsula), the total area is of $22,446 \mathrm{~km}^{2}$. This region ranks sixth in Italy. The region comprises of plains (48\%), hills (27\%) and mountains (25\%) with an altitude of more than 2,000 m above mean sea level. In 2019, the population of the region was estimated to be 4,459,577 (ISTAT 2011, http://dati.istat.it/) with a population density of approximately 200 inhabitants per km². 


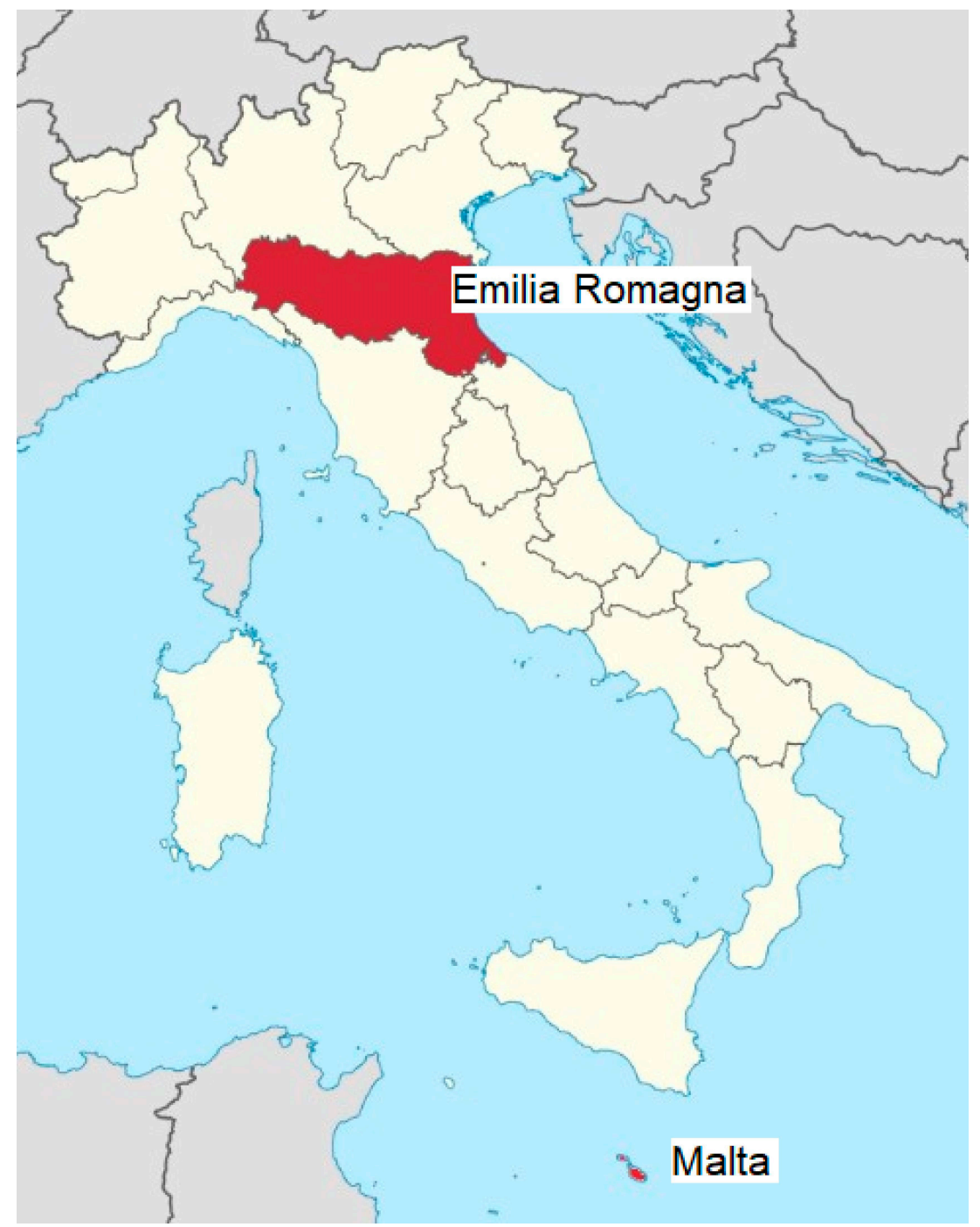

Figure 1. The Emilia Romagna region (Italy) and the Maltese Islands

\section{Data collection}

Ethnobotanical data was collected from several sources related to the two respective regions. For this research, information about medicinal plants usages was found in books and scientific articles published in journals and available on online platforms. Usages and traditions related to Emilia Romagna were researched in the library of Agriculture of Bologna (Lazzarini 1992 and 1996, Lanzara 1978, Pignatti 2003). On the other hand, in Malta, the books regarding this topic were mainly placed in the "Melitensia special collection", a particular section of the main library of the University. Some of the books that have been used, in fact, were old and without an English version (Lanfranco 1993, Penza 1969). Consequently, the information was translated from Maltese to English, with the help of some Maltese people. More recent studies conducted on the Maltese flora were also considered (Attard \& Cuschieri 2009, Attard \& Pacioni 2012, Attard et al. 2015, Caruana \& Attard 2016). The first step was the compilation of the list of plants that were common to both regions omitting the others that occurred in only one of the regions.

\section{Calculations and data analysis}

Following the categorization of the plant species by family, the uses of each plant in the respective location were listed and transformed numerically to determine the popular therapeutic ratio, as a modification to the equation used by Bulut and Tuzlaci (2015), taken as number of common therapeutic uses/total number of therapeutic uses. The popularity index was determined from the graphs obtained for relationships between the two regions. Pearson correlation followed by Principal Component Analysis were conducted by plant family and by use. Statistical significance was considered at $p<0.05$. 


\section{Results and discussion \\ Plant families with ethnobotanical uses}

The Maltese flora is made up of approximately 1264 vascular plants with 458 species used in folk medicine for the treatment of several diseases (Lanfranco 1975, 1993). In this case, $42 \%$ of the medicinal species were considered. On the other hand, within the Emilia-Romagna region, there are 3927 taxa (https://bbcc.ibc.regione.emiliaromagna.it/pater/search.do?type=bnb\&page=1\&re=load) with about 500 species used traditionally for medicinal purposes. This number is not precise, although realistic as it derives from bibliographic sources (Lazzarini 1992, Lazzarini 1996) and information obtained from the "Giardino delle erbe officinali Augusto Rinaldi Ceroni, Casola Valsenio (RA)", which collects, preserves, and cultivates plants of medicinal and aromatic interests and belongs to the Emilia-Romagna region (https://ilgiardinodelleerbe.it/). For this region, 39\% of the medicinal species were considered in the present study. Table 1 represents a comparison of the ethnobotanical particulars of two regions. The Maltese region shows a medicinal plant density of 1.45 per $\mathrm{km}^{2}$ whereas the Emilia-Romagna region has a density of 0.02 per $\mathrm{km}^{2}$. The density of the medicinal flora within the two regions compares with the minimum and maximum densities within the Pyrenees region (min: 0.035, max: 1.68 per km²) (Akerreta et al. 2007), but the Maltese medicinal flora density is almost three times higher than that of the eastern Mallorca region $\left(0.51\right.$ per $\left.\mathrm{km}^{2}\right)(\mathrm{Carrio}$ \& Valles 2012).

Table 1. Comparison of ethnobotanical particulars of two regions.

\begin{tabular}{|l|l|l|l|l|l|}
\hline Region & Extension $\left(\mathrm{km}^{2}\right)$ & Population & Flora & $\mathrm{MP}$ & $\mathrm{MP} / \mathrm{km}^{2}$ \\
\hline Emilia-Romagna & 22,446 & $4,459,577$ & 3927 & 500 & 0.02 \\
\hline Malta & 316 & 515,000 & 1264 & 458 & 1.45 \\
\hline
\end{tabular}

\section{Species from the Asteraceae, Lamiaceae and Apiaceae plant families}

Following a thorough search for sources, the number of species in common between the two regions was 193 (Table 2). These species were distributed in 72 plant families. Due to the long list of species three plant families having the highest number of species were studied further. These include the Asteraceae, Lamiaceae and Apiaceae families (10.4\%, $7.8 \%$ and $5.2 \%$, respectively). All the other families had on average approximately $0.5-2 \%$ of species. To some extent, this goes in accordance with other studies. Akerrata and co-workers (2007), Hadjichambis and co-workers (2008) and Nawash and co-workers (2014) reported that the predominating botanical family was Asteraceae, considering that this family is well represented in the flora of several Mediterranean countries. Others quoted the Lamiaceae as the predominating botanical family (Novais et al. 2004; González-Tejero et al. 2008; Carrio \& Valles 2012; Bulut et al. 2017). Whereas the studies mentioned above, listed the Asteraceae or Lamiaceae family in second place, only Rivera and co-workers (2005) quoted Apiaceae in the third position as reported in this current study. As stated by several authors (Johns et al. 1990; Bonet et al. 1999; Agelet \& Valles 2001; Novais et al. 2004), the relative abundance of a plant reflects the extent of its potential popular use.

Table 2. List of the common medicinal species present in Malta and Emilia Romagna

\begin{tabular}{|l|l|}
\hline Family & Species \\
\hline Acanthaceae & Acanthus mollis \\
\hline Alismaceae & Alisma plantago aquatica \\
\hline Amaranthaceae & Amaranthus blitum, Amaranthus graecizans, Amaranthus retroflexus \\
\hline Ampelidaceae & Vitis vinifera \\
\hline Anacardiaceae & Rhus coriari, Schinus mollea \\
\hline Apiaceae & $\begin{array}{l}\text { Amni majus, Amni visnaga, Anethum graveolens, Anthriscus cerefolium, Apium graveolens, } \\
\text { Carum carvi, Conium maculatum, Coriandrum sativum, Daucus carota, Foeniculum vulgare, } \\
\text { Cuminum cyminum, Petroselinum crispum, Oenanthe acquatica, Pimpinella anisum }\end{array}$ \\
\hline Apocynaceae & Vinca major \\
\hline Araceae & Arum italicum, Arum maculatum \\
\hline Araliaceae & Hedera helix \\
\hline Asteraceae & $\begin{array}{l}\text { Achillea millefolium, Anthemis arvensis, Artemisa campestris, Artemisia alba, Artemisia } \\
\text { dracunculus, Bellis perennis, Calendula arvensis, Calendula officinalis, Carlina vulgaris, } \\
\text { Centaurea calcitrapa, Carthamus tinctorius, Cichorium intybus, Cnicus benedictus, Cynara } \\
\text { scolymus, Eupatorium cannabium, Helianthus tuberosus, Helicrysum italicum, Inula }\end{array}$ \\
\hline
\end{tabular}




\begin{tabular}{|c|c|}
\hline & $\begin{array}{l}\text { graveolens, Lactuca saligna, Lactuca virosa, Lavandula angustifolia, Matricaria chamomilla, } \\
\text { Pulicaria dysenterica, Santolina chamaecyparissus, Senecio vulgaris, Sonchus asper, } \\
\text { Taraxacum officinale, Tragopogon porrifolius, Xanthium spinosum }\end{array}$ \\
\hline Boraginaceae & $\begin{array}{l}\text { Anchusa italica, Borago officinalis, Cynoglossum creticum, Echium vulgare, Symphytum } \\
\text { officinale }\end{array}$ \\
\hline Brassicacae & Eruca vesicaria \\
\hline Buxaceae & Buxus sempervirens \\
\hline Capparidaceae & Capparis spinosa \\
\hline Caprifoliaceae & Lonicera caprifolium, Sambucus ebulus, Sambucus nigra \\
\hline Caryophyllaceae & Arenaria serpyllifolia, Hernaria glabra, Silene vulgaris, Stellaria media \\
\hline Chenopodiaceae & Beta vulgaris \\
\hline Chenopodiaceae & Chenopodium album \\
\hline Ciperaceae & Cyperus longus \\
\hline Convolvulaceae & Convolvulus arvensis, Cuscuta epithymum \\
\hline Crucifereae & $\begin{array}{l}\text { Brassica napus, Brassica oleracea, Brassica rapa ssp. campestris, Capsella bursa pastoris, } \\
\text { Cardamine hirsuta, Cheiranthus cheiri, Diplotaxis muralis, Lepidum sativum, Nasturtium } \\
\text { officinale, Raphanus raphanistrum, Sinapis alba, Sisymbrium officinale }\end{array}$ \\
\hline Cucurbitaceae & Ecballium elaterium \\
\hline Cupressaceae & Cupressus sempervirens \\
\hline Cupulifereae & Corylus avellana \\
\hline Cyperaceae & Scirpus lacustris \\
\hline Dioscoreaceae & Tamus communis \\
\hline Dipsacaceae & Dipsacus sylvestris \\
\hline Dryopteridaceae & Dryopteris filix-mas \\
\hline Equisetaceae & Equisetum ramosissimum \\
\hline Ericaceae & Arbutus unedo \\
\hline Euphorbiaceae & Euphorbia helioscopia \\
\hline Euphorbiaceae & Mercurialis annua \\
\hline Fagaceae & Quercus ilex \\
\hline Fumariaceae & Fumaria capreolata, Fumaria officinalis \\
\hline Geraniaceae & Erodium cicutarium, Geranium robertianum \\
\hline Ginkgoaceae & Ginkgo biloba \\
\hline Gramineae & Agropyrum repens, Arundo donax, Avena fatua, Avena sativa, Cynodon dactylon \\
\hline Hyperiaceae & Hypericum perforatum \\
\hline Iridaceae & Gladiolus italicus, Iris germanica \\
\hline Juglandaceae & Juglans regia \\
\hline Lamiaceae & $\begin{array}{l}\text { Ajuga reptans, Ballota nigra, Glechoma hederacea, Marrubium vulgare, Melissa officinalis, } \\
\text { Mentha arvensis, Mentha pulegium, Mentha rotundifolia, Ocimum basilicum, Origanum } \\
\text { majorana, Origanum vulgare, Rosmarinus officinalis, Salvia officinalis, Thymus vulgaris }\end{array}$ \\
\hline Lauraceae & Laurus nobilis \\
\hline Leguminosae & $\begin{array}{l}\text { Anthyllis vulneraria, Lotus corniculatus, Medicago sativa, Ononis spinosa, Robinia } \\
\text { pseudoacacia, Spartium junceum, Trifolium pratense, Trifolium repens, Trigonella foenum } \\
\text { graecum }\end{array}$ \\
\hline Liliaceae & $\begin{array}{l}\text { Allium sativum, Asparagus acutifolius, Asparagus officinalis, Colchium autumnale, Muscari } \\
\text { comosum, Ruscus aculeatos, Smilax aspera }\end{array}$ \\
\hline Linaceae & Linum usitatissimum \\
\hline Lythraceae & Lythrum hyssopifolia \\
\hline Malvaceae & Althea officinalis, Malva rotundifolia, Malva sylvestris \\
\hline Moraceae & Ficus carica, Morus alba, Morus nigra \\
\hline Nyctaginaceae & Mirabilis jalapa \\
\hline Nymphaeaceae & Nymphaea alba \\
\hline Oleaceae & Fraxinus excelsior, Fraxinus ornus, Olea europea \\
\hline Orchideaceae & Orchis morio \\
\hline Papaveraceae & Chelidonium majus, Papaver rhoeas, Papaver somniferum \\
\hline Pinaceae & Pinus halepensis \\
\hline
\end{tabular}




\begin{tabular}{|l|l|}
\hline Poaceae & Hordeum vulgare \\
\hline Polygonaceae & $\begin{array}{l}\text { Polygonium aviculare, Polygonum lapathifolium, Rumex acetosa, Adiantum capillus veneris, } \\
\text { Asplenium trichomanes, Ceterach officinarium }\end{array}$ \\
\hline Portulacaceae & Portulaca oleracea \\
\hline Primulaceae & Anagallis arvensis \\
\hline Punicaceae & Punica granatum \\
\hline Phytolaccaceae & Phytolacca decandra \\
\hline Ranuncolaceae & Anemone coronaria, Clematis vitalba, Ranunculus arvensis \\
\hline Rosaceae & $\begin{array}{l}\text { Crataegus monogyna, Fragaria vesca, Mespilus germanica, Potentilla reptans, Prunus } \\
\text { domestica, Rosa canina, Rubus ulmifolius, Sanguisorba minor }\end{array}$ \\
\hline Rubiaceae & Asperula cynanchica, Galium aparine \\
\hline Rutaceae & Ruta graveolens \\
\hline Salicacae & Populus alba, Salix alba \\
\hline Scrophulariaceae & $\begin{array}{l}\text { Anthirrinum majus, Scophularia nodosa, Verbascum thapsus, Verbascum thapsus, Veronica } \\
\text { anagallis-aquatica }\end{array}$ \\
\hline Simarubaceae & Ailanthus altissima \\
\hline Solanaceae & Atropa belladonna, Datura stramonium, Physalis alkekengi, Solanum nigrum \\
\hline Typhaceae & Typha angustifolia, Typha latifolia \\
\hline Urticaceae & Parietaria officinalis, Urtica urens \\
\hline Valerianaceae & Centranthus ruber, Valeriana officinalis \\
\hline Verbenaceae & Lippia citriodora, Verbena officinalis, Vitex agnus-castus \\
\hline Violaceae & Viola odorata, Viola tricolor \\
\hline Zygophyllaceae & Tribulus terrestris \\
\hline
\end{tabular}

\section{Asteraceae family}

Table 3 represents the uses of the eighteen species within the two regions, together with the popular therapeutic uses and the respective ratio. It was observed that the popular therapeutic ratio for Anthemis arvensis, Bellis perrenis, Inula graveolens and Tragopogon porrifolius was zero, indicating that there were no common traditional uses for the two regions. On the other hand, in the case of Carthamus tinctorius and Helianthus tuberosus the popular therapeutic ratio was 1 , indicating that these species were used for the same ailments in both regions. Figure 2 illustrates the popularity of Asteraceae plants by use. It is clearly indicated that six out of the eighteen species had a high popularity index in both regions, these include Artemisia absinthium, Cichorium intybus, Taraxacum officinale, Cynara scolymus, Matricaria chamomilla and Achillea millefolium. Despite a variation in the popular therapeutic ratio $(0.14-1.00)$, these Asteraceae herbs are popular in both regions. Novais and co-workers (2004) mentioned, Anthemis nobilis as the most common Asteaceae species of Arrabida Natural Park (Portugal). Maxia and co-workers (2008) considered Inula viscosa and Centaurea calcitrapa as two important species in southwestern Sardinia.

Table 3. The uses of the eighteen Asteraceae species within the two regions

\begin{tabular}{|c|c|c|c|}
\hline Asteraceae & MT & ER & $\begin{array}{l}\text { Popular } \\
\text { therapeutic } \\
\text { ratio }\end{array}$ \\
\hline Achillea millefolium $\mathrm{L}$. & $\begin{array}{l}\text { antispasmodic, digestive, } \\
\text { cholagogue, emmenagogue, } \\
\text { antidiarrhoeic, anti- } \\
\text { haemorrages, depurative, skin } \\
\text { injuries and excoriations, } \\
\text { increase appetite, surface veins }\end{array}$ & $\begin{array}{l}\text { antispasmodic, digestive, } \\
\text { cholagogue, emmenagogue, } \\
\text { antidiarrhoeic, anti- } \\
\text { haemorrages, depurative, skin } \\
\text { injuries and excoriations, for } \\
\text { toothache }\end{array}$ & 0.73 \\
\hline Anthemis arvensis $\mathrm{L}$. & stomachache, skin itching & $\begin{array}{l}\text { antispasmodic, digestive, } \\
\text { antipyrethic }\end{array}$ & 0.00 \\
\hline Artemisia absinthium $\mathrm{L}$. & $\begin{array}{l}\text { antihelmintic, antimicrobial and } \\
\text { antiseptic, insect repellent, anti- } \\
\text { asthmatic, for hair, skin } \\
\text { problems, toothache, }\end{array}$ & $\begin{array}{l}\text { antihelmintic, antiinflammatory, } \\
\text { antimicrobial and antiseptic, } \\
\text { antidepressant, digestive, } \\
\text { carminative }\end{array}$ & 0.14 \\
\hline
\end{tabular}




\begin{tabular}{|c|c|c|c|}
\hline & $\begin{array}{l}\text { cholagogue, emmenagogue, } \\
\text { eyes }\end{array}$ & & \\
\hline Bellis perennis L. & stomachic, coughs & $\begin{array}{l}\text { rheumatism, wounds and } \\
\text { inflammation, diaphoretic, } \\
\text { laxative }\end{array}$ & 0.00 \\
\hline Calendula spp. & $\begin{array}{l}\text { antispasmodic, jaundice, } \\
\text { chilblains, heart problems }\end{array}$ & $\begin{array}{l}\text { antispasmodic, emmenagogue, } \\
\text { antibacterial and antifungal } \\
\text { activity, sedative, hypertension, } \\
\text { skin lesions, insect bites, } \\
\text { erythema }\end{array}$ & 0.13 \\
\hline Carthamus tinctorius $\mathrm{L}$. & laxative & laxative & 1.00 \\
\hline Centaurea calcitrapa L. & antipyretic & $\begin{array}{l}\text { carminative, digestive, diuretic, } \\
\text { antipyretic }\end{array}$ & 0.25 \\
\hline Cichorium intybus L. & $\begin{array}{l}\text { diuretic, digestive, stomachic, } \\
\text { cholagogue, diabetes, } \\
\text { antihaemorroids, aphrodisiac, } \\
\text { skin problems }\end{array}$ & $\begin{array}{l}\text { depurative, tonic, antipyretic, } \\
\text { diuretic, digestive, stomachic }\end{array}$ & 0.27 \\
\hline Cynara scolymus L. & $\begin{array}{l}\text { diuretic, cholagogue, tonic, } \\
\text { antiphyretic, diabetes, } \\
\text { hypocholesterolaemic, } \\
\text { rheumatism }\end{array}$ & $\begin{array}{l}\text { diuretic, depurative, digestive, } \\
\text { cholagogue, diabetes, } \\
\text { hypocholesterolaemic }\end{array}$ & 0.44 \\
\hline $\begin{array}{l}\text { Eupatorium cannabium } \\
\text { L. }\end{array}$ & $\begin{array}{l}\text { laxative, diuretic, cholagogue, } \\
\text { for colds, diaphoretic, insect } \\
\text { repellent }\end{array}$ & laxative, diuretic, cholagogue & 0.50 \\
\hline Helianthus tuberosus L. & diabetes & diabetes & 1.00 \\
\hline $\begin{array}{l}\text { Helichrysum italicum } \\
\text { (Roth) G. Don fil. }\end{array}$ & $\begin{array}{l}\text { antiasthmatic and for } \\
\text { respiratory system }\end{array}$ & $\begin{array}{l}\text { cough, colds and allergies, } \\
\text { antiasthmatic and for respiratory } \\
\text { system, headaches, liver } \\
\text { problems, arthosis, skin } \\
\text { problems, burns, depurative, } \\
\text { diaphoretic }\end{array}$ & 0.13 \\
\hline $\begin{array}{l}\text { Inula graveolens }(\mathrm{L} .) \\
\text { Desf. }\end{array}$ & insect repellent & $\begin{array}{l}\text { stomachic, cholagogue, cough, } \\
\text { diaphoretic }\end{array}$ & 0.00 \\
\hline Lactuca virosa $\mathrm{L}$. & sedative & sedative, emollient, hypnotic & 0.33 \\
\hline Matricaria chamomilla L. & $\begin{array}{l}\text { stomachic, increase appetite, } \\
\text { ulcers, antispasmodic, } \\
\text { antiseptic, laxative, insomnia, } \\
\text { skin problems, surface veins, } \\
\text { eye inflammation }\end{array}$ & $\begin{array}{l}\text { stomachic, headaches, } \\
\text { antispasmodic, antiseptic, } \\
\text { laxative, insomnia, skin } \\
\text { problems, diaphoretic }\end{array}$ & 0.50 \\
\hline Senecio vulgaris $\mathrm{L}$. & $\begin{array}{l}\text { sedative, emmenagogue, } \\
\text { mouth problems }\end{array}$ & $\begin{array}{l}\text { sedative, emmenagogue, } \\
\text { purgative and antihelmintic (but } \\
\text { cirrhosis) }\end{array}$ & 0.50 \\
\hline $\begin{array}{l}\text { Taraxacum officinale (L.) } \\
\text { Weber ex F.H.Wigg. }\end{array}$ & $\begin{array}{l}\text { increase appetite, digestive, } \\
\text { cholagogue, depurative, } \\
\text { laxative, diuretic, sedative, } \\
\text { headaches }\end{array}$ & $\begin{array}{l}\text { increase appetite, digestive, } \\
\text { cholagogue, depurative, laxative, } \\
\text { diuretic }\end{array}$ & 0.75 \\
\hline $\begin{array}{l}\text { Tragopogon porrifolius } \\
\text { L. }\end{array}$ & coughs, skin problems & $\begin{array}{l}\text { depurative, stomachic, } \\
\text { astringent, metabolism booster }\end{array}$ & 0.00 \\
\hline
\end{tabular}




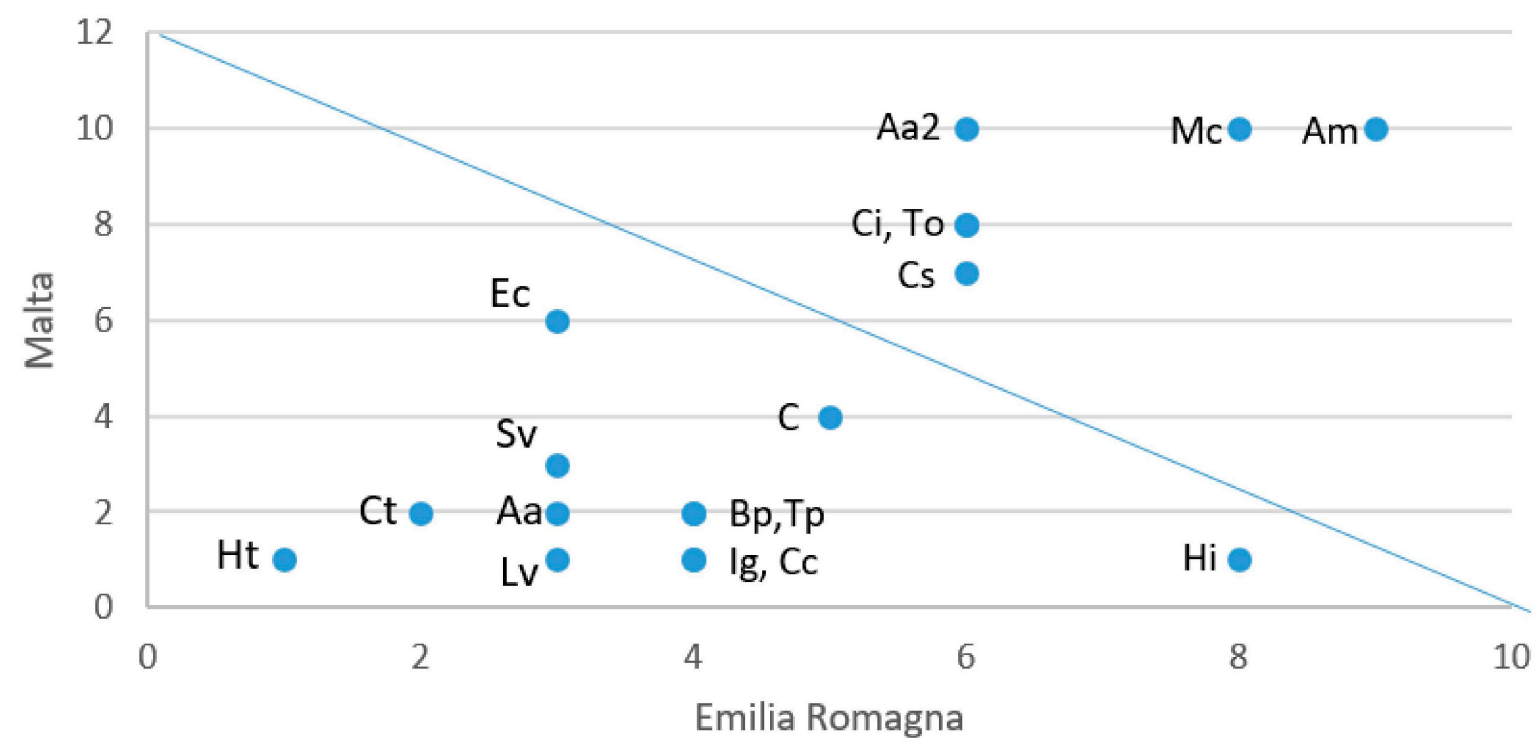

Figure 2. Asteraceae: Popularity by Use within the two regions

\section{Lamiaceae family}

Table 4 illustrates the uses of eleven species within the two regions. Within this group, none of the species scored zero with regards to the popular therapeutic ratio, indicating that there is a degree of commonality between the uses of these species within the two regions. On the other hand, none of the species had a popular therapeutic ratio of 1 . The species with the lowest score was Ocimum basilicum $(0.17)$ and the one with the highest score was Thymus vulgaris (0.82). Figure 3 shows the popularity index for the species, indicating a high popularity index for nine out of the eleven species. These include Ballota nigra/Marrubium vulgare, Lavandula angustifolia, Mentha spp., Melissa officinalis, Origanum spp., Rosmarinus officinalis, Salvia officinalis, and Thymus vulgaris. Despite a variation in the popular therapeutic ratio $(0.31-0.82)$, these Lamiaceae herbs are popular in both regions. Menthais amongst the predominating genera in Egypt, Morocco, and Spain, Origanum vulgare in Albania, Rosmarinus officinalis in Algeria and Salvia officinalis in Albania (González-Tejero et al. 2008). Novais and co-workers (2004) mentioned, Melissa officinalis, Mentha pulegium, Phlomis purpurea and Rosmarinus officinalis as the most common Lamiaceae species of Arrabida Natural Park (Portugal).

Table 4. The uses of the eleven Lamiaceae species within the two regions

\begin{tabular}{|c|c|c|c|}
\hline Lamiaceae & MT & ER & $\begin{array}{l}\text { Popular } \\
\text { therapeutic } \\
\text { ratio }\end{array}$ \\
\hline Ajuga reptans $\mathrm{L}$. & $\begin{array}{l}\text { astringent, antidiarrhoeic, skin } \\
\text { problems, toothache and sore } \\
\text { throat, arthritis, rheumatism }\end{array}$ & $\begin{array}{l}\text { astringent, antidiarrhoeic, skin } \\
\text { problems, toothache and sore } \\
\text { throat }\end{array}$ & 0.67 \\
\hline $\begin{array}{l}\text { Ballota nigra L. / } \\
\text { Marrubium vulgare L. }\end{array}$ & $\begin{array}{l}\text { digestive, antispasmodic, } \\
\text { cholagogue, coughs, viral } \\
\text { diseases, skin problems, } \\
\text { metabolism booster, eye and } \\
\text { ears conditions, rheumatism, } \\
\text { antipyretic }\end{array}$ & $\begin{array}{l}\text { digestive, antispasmodic, } \\
\text { cholagogue, emmenagogue, } \\
\text { sedative, coughs, diaphoretic }\end{array}$ & 0.31 \\
\hline Glechoma hederacea L. & $\begin{array}{l}\text { astringent, antidiarrhoeic, } \\
\text { haemorrohids, respiratory } \\
\text { system }\end{array}$ & $\begin{array}{l}\text { astringent, antidiarrhoeic, } \\
\text { haemorrohids, respiratory } \\
\text { system, diuretic }\end{array}$ & 0.80 \\
\hline $\begin{array}{l}\text { Lavandula angustifolia } \\
\text { Mill. }\end{array}$ & $\begin{array}{l}\text { sedative, cholagogue, } \\
\text { antispasmodic, antidiharroeic, } \\
\text { carminative, insect repellent, } \\
\text { antiseptic }\end{array}$ & $\begin{array}{l}\text { sedative, cholagogue, } \\
\text { antispasmodic, antidiharroeic, } \\
\text { carminative, insect repellent, } \\
\text { antiseptic, rheumatism, coughs }\end{array}$ & 0.78 \\
\hline Melissa officinalis L. & $\begin{array}{l}\text { sedative (for insomnia and } \\
\text { stress), antispasmodic, } \\
\text { carminative, emmenagogue, } \\
\text { cicatrising role, } \\
\text { antiinflammatory, joint and }\end{array}$ & $\begin{array}{l}\text { sedative (for insomnia and } \\
\text { stress), antispasmodic, } \\
\text { carminative, emmenagogue, } \\
\text { cicatrising role, }\end{array}$ & 0.70 \\
\hline
\end{tabular}




\begin{tabular}{|c|c|c|c|}
\hline & $\begin{array}{l}\text { muscular pain relief, hair loss, } \\
\text { antipyretic, psoriasis }\end{array}$ & $\begin{array}{l}\text { antiinflammatory, joint and } \\
\text { muscular pain relief }\end{array}$ & \\
\hline Mentha spp. & $\begin{array}{l}\text { stomachache, antidiarrhoeic, } \\
\text { antispasmodic, tonic, against } \\
\text { liver problems and jaundice, } \\
\text { rheumatism, sedative and to } \\
\text { treat shocks, antiseptic, insect } \\
\text { repellent }\end{array}$ & $\begin{array}{l}\text { stomachache, antidiarrhoeic, } \\
\text { antispasmodic, tonic, against } \\
\text { liver problems and jaundice, } \\
\text { rheumatism }\end{array}$ & 0.67 \\
\hline Ocimum basilicum L. & $\begin{array}{l}\text { insect repellent, antipyretic, } \\
\text { kidney problems }\end{array}$ & $\begin{array}{l}\text { antispasmodic, sedative, skin } \\
\text { irritation, insect repellent }\end{array}$ & 0.17 \\
\hline Origanum spp. & $\begin{array}{l}\text { digestive, stomachic, } \\
\text { carminative, tonic (stimulant), } \\
\text { antispasmodic, diaphoretic, } \\
\text { headache, expectorant, } \\
\text { cholagogue, emmenagogue, } \\
\text { antiseptic }\end{array}$ & $\begin{array}{l}\text { digestive, stomachic, } \\
\text { carminative, tonic (stimulant), } \\
\text { antispasmodic, expectorant, } \\
\text { sedative, antiseptic }\end{array}$ & 0.58 \\
\hline Rosmarinus officinalis L. & $\begin{array}{l}\text { digestive, cholagogue, } \\
\text { emmenagogue, coughs, } \\
\text { diaphoretic, sedative, joint and } \\
\text { muscular pain relief, antiseptic, } \\
\text { tonic, circulation, hair, } \\
\text { antispasmodic }\end{array}$ & $\begin{array}{l}\text { digestive, cholagogue, diuretic, } \\
\text { emmenagogue, coughs, } \\
\text { diaphoretic, joint and muscular } \\
\text { pain relief, antiseptic, tonic, } \\
\text { circulation, hair, insect repellent }\end{array}$ & 0.71 \\
\hline Salvia officinalis L. & $\begin{array}{l}\text { digestive, cholagogue, diuretic, } \\
\text { diaphoretic, stop milk } \\
\text { production, anti-asthmatic, } \\
\text { antiseptic, skin problems }\end{array}$ & $\begin{array}{l}\text { digestive, cholagogue, diuretic, } \\
\text { diaphoretic, stop milk } \\
\text { production, anti-asthmatic, } \\
\text { antiseptic, skin problems, } \\
\text { cicatrising role, mouth problems, } \\
\text { hair }\end{array}$ & 0.73 \\
\hline Thymus vulgaris L. & $\begin{array}{l}\text { carminative, diaphoretic, } \\
\text { depurative, emmenagogue, } \\
\text { antidiarrhoeic, antispasmodic, } \\
\text { antiseptic, skin (wounds and } \\
\text { plagues), mouth problems, } \\
\text { arthritis pain, headaches }\end{array}$ & $\begin{array}{l}\text { carminative, diaphoretic, } \\
\text { depurative, emmenagogue, } \\
\text { antidiarrhoeic, antispasmodic, } \\
\text { antiseptic, skin (wounds and } \\
\text { plagues), mouth problems }\end{array}$ & 0.82 \\
\hline
\end{tabular}

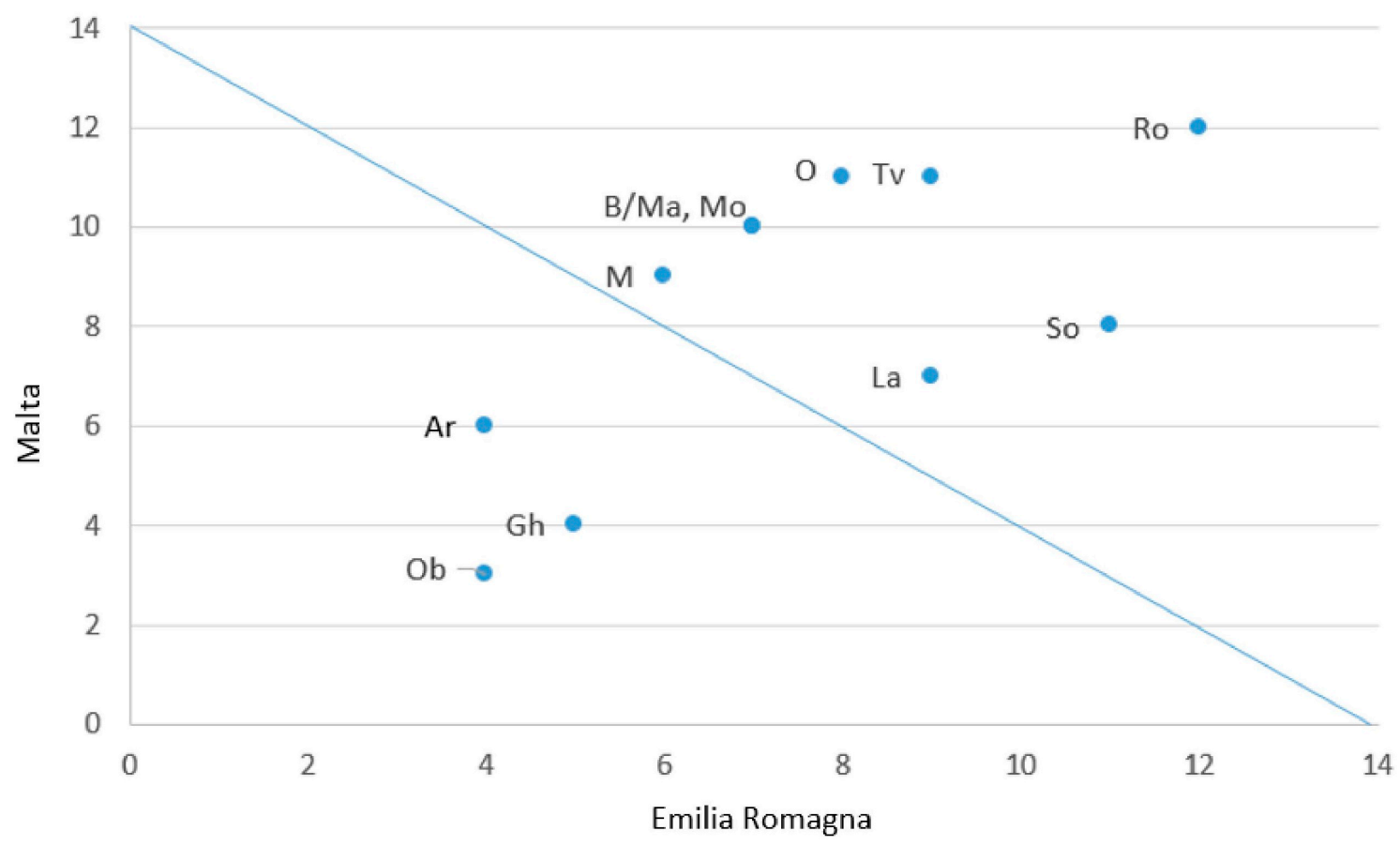

Figure 3. Lamiaceae: Popularity by use within the two regions 


\section{Apiaceae family}

Table 5 represents the uses of nine species from the Apiaceae family within the two regions. Although all nine species are present in both regions, two species were unpopular in one of the two regions. Coriandrum sativum showed three uses in the Emilia-Romagna region but one medicinal use in Malta, while Cuminum cyminum had two medicinal uses in Malta, but one in the Emilia-Romagna region, with no overlaps for both species. The highest popular therapeutic ratio was exhibited by Ammi majus (0.60), whilst the lowest by Foeniculum vulgare $(0.27)$. Figure 4 illustrates that only two out of the nine species had a high the popularity index, and these are Foeniculum vulgare and Daucus carota. However, both species exhibited a low popular therapeutic ratio $(0.27$ and 0.44 , respectively), when compared between the two regions.

Table 5. The uses of the nine Apiaceae species within the two regions

\begin{tabular}{|c|c|c|c|}
\hline Apiaceae & MT & ER & $\begin{array}{l}\text { Popular } \\
\text { therapeutic } \\
\text { ratio }\end{array}$ \\
\hline Amni majus L. & $\begin{array}{l}\text { diuretic, carminative, } \\
\text { stomachic, tonic, antiasthmatic }\end{array}$ & diuretic, carminative, stomachic & 0.60 \\
\hline Anethum graveolens $\mathrm{L}$. & $\begin{array}{l}\text { carminative, stomachic, } \\
\text { digestive, sedative, } \\
\text { haemorrhoids }\end{array}$ & $\begin{array}{l}\text { carminative, stomachic, } \\
\text { digestive, galactagogue }\end{array}$ & 0.50 \\
\hline Apium graveolens $\mathrm{L}$. & $\begin{array}{l}\text { carminative, diuretic, } \\
\text { rheumatism, antiseptic (urinary } \\
\text { tract), aphrodisiac }\end{array}$ & carminative, diuretic & 0.40 \\
\hline Carvum carvi $\mathrm{L}$. & $\begin{array}{l}\text { carminative, digestive, skin } \\
\text { problems ( itching and } \\
\text { parasites) }\end{array}$ & $\begin{array}{l}\text { carminative, digestive, } \\
\text { galactagogue, diuretic }\end{array}$ & 0.40 \\
\hline Coriandrum sativum $\mathrm{L}$. & carminative & $\begin{array}{l}\text { antispasmodic, diaphoretic, } \\
\text { digestive }\end{array}$ & 0.00 \\
\hline Cuminum cyminum $\mathrm{L}$. & $\begin{array}{l}\text { increase appetite, high } \\
\text { pressure }\end{array}$ & carminative & 0.00 \\
\hline Daucus carota L. & $\begin{array}{l}\text { eyes, diuretic, depurative, } \\
\text { stomachic, antihelmintic }\end{array}$ & $\begin{array}{l}\text { eyes, diuretic, depurative, } \\
\text { stomachic, antidiarrhoeic for } \\
\text { infants, galactagogue, } \\
\text { carminative, emmenagogue }\end{array}$ & 0.44 \\
\hline Foeniculum vulgare Mill. & $\begin{array}{l}\text { carminative, digestive, eyes, } \\
\text { throat infections, diuretic, } \\
\text { galactagogue, emmenagogue, } \\
\text { insect repellent, pain killer, skin } \\
\text { problems }\end{array}$ & $\begin{array}{l}\text { carminative, digestive, } \\
\text { antispasmodic, diaphoretic, } \\
\text { galactagogue, mmenagogue, } \\
\text { depigmentant on skin, tonic in } \\
\text { bath, expectorant }\end{array}$ & 0.27 \\
\hline $\begin{array}{l}\text { Petroselinum crispum } \\
\text { (Mill.) Fuss/Anthriscus } \\
\text { cerefolium (L.) Hoffm. }\end{array}$ & $\begin{array}{l}\text { diuretic, carminative, } \\
\text { depurative, emmenagogue, } \\
\text { skin problems, chest irritation } \\
\text { in lactating mothers, } \\
\text { aphrodisiac }\end{array}$ & $\begin{array}{l}\text { diuretic, carminative, depurative, } \\
\text { emmenagogue }\end{array}$ & 0.57 \\
\hline
\end{tabular}

\section{Therapeutic Systems}

Table 6 represents the number of uses by therapeutic systems for the two regions. Each system represented several ailments. The heart and circulatory system were represented by hyper-cholesterolaemia, hypertension, jaundice, heart problems, varicose veins, chilblains, bleeding, and circulatory problems. The central nervous system was represented by sedation, antidepressant, and headaches, while the urinary system was represented by diuresis. The respiratory system was presented by asthma, dry and productive coughs, colds, respiratory allergies. The skin, nails and hair system were represented by insect bites, skin lesions, erythema, skin burns, scar formation, hair problems, psoriasis, and skin irritation. The musculoskeletal system was represented by arthritis, rheumatic disorders, joint and muscular pain, while the endocrine system was represented by diabetes, diaphoresis, pyrexia, and milk production disorders in nursing mothers. The reproductive system was represented by menstrual disorders and aphrodisiac. The gastrointestinal system was represented by loss of appetite, stomach upsets, gastric ulceration, liver and biliary problems, diarrhea, intestinal spasms, intestinal worms, and constipation.

In Table 6, it is clearly shown that the uses of herbs from the Asteraceae, Lamiaceae and Apiaceae families, are prominent towards gastrointestinal ailments, with an overall score of 89 and 77 for the Emilia-Romagna region and Malta, respectively. The same family ranking was observed in other studies (Bhardwaj et al. 2019). Conditions of the 
musculoskeletal system and the eyes/ears/mouth origin were the least treated in these two regions ( 6 and 8, ER and MT; 4 and 10, ER and MT, respectively). Comparing the three plant families for the treatment of ailments, the overall decreasing ranking order by use is Lamiaceae, Asteraceae and Apiaceae (173, 163 and 81, respectively).

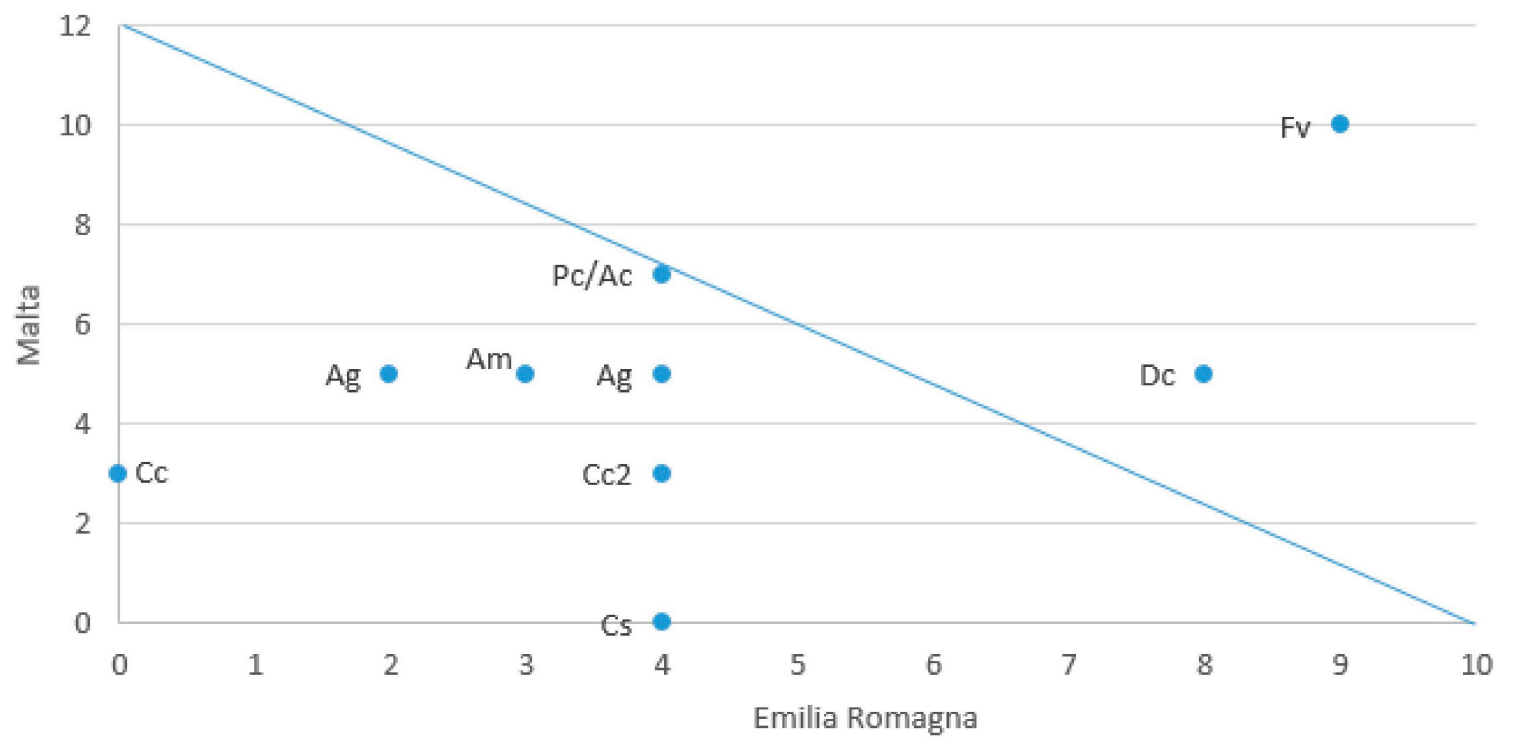

Figure 4. Apiaceae: Popularity by use within the two regions

Table 6. The number of uses by therapeutic systems for the three families within the two regions

\begin{tabular}{|l|c|c|c|c|c|c|}
\hline \multirow{2}{*}{ By system } & \multicolumn{2}{|c|}{ Asteraceae } & \multicolumn{2}{c|}{ Lamiaceae } & \multicolumn{2}{c|}{ Apiaceae } \\
\cline { 2 - 7 } & MT & ER & MT & ER & MT & ER \\
\hline Circulatory System and Heart & 8 & 3 & 2 & 2 & 2 & 0 \\
\hline Central Nervous System and Brain & 5 & 8 & 6 & 5 & 1 & 0 \\
\hline Urinary System & 4 & 5 & 2 & 3 & 5 & 5 \\
\hline Gastrointestinal Tract & 27 & 39 & 31 & 30 & 19 & 20 \\
\hline Respiratory System & 4 & 3 & 5 & 6 & 0 & 1 \\
\hline Skin, Nails and Hair & 7 & 3 & 8 & 9 & 4 & 1 \\
\hline Eyes Ears and Mouth & 4 & 1 & 3 & 2 & 3 & 1 \\
\hline Musculoskeletal System & 1 & 2 & 6 & 4 & 1 & 0 \\
\hline Endocrine System & 6 & 8 & 8 & 5 & 1 & 6 \\
\hline Reproductive System & 4 & 3 & 4 & 4 & 4 & 3 \\
\hline For all the systems (eg. Antiseptic) & 7 & 11 & 15 & 13 & 3 & 1 \\
\hline Total by region and family & 77 & 86 & 83 & 90 & 43 & 38 \\
\hline Total by family & & & & 173 & & 81 \\
\hline
\end{tabular}

\section{Correlations by Uses and Therapeutic Systems}

In order to determine the significance of correlations between the two regions in terms of herb uses and therapeutic systems, the data was subjected to Pearson's correlation and Principal Component Analysis (PCA). The correlation between the two regions for the Asteraceae, Lamiaceae and Apiaceae families was $0.953,0.987$ and 0.927 , respectively. PCA by family (Figure 5a) shows that the two regions were clustered for each family. Species from the Asteraceae family showed the highest uses for most systems (particularly the CNS and Gl system), whereas Lamiaceae scored second and Apiaceae showed the lowest uses except for the urinary system. This shows that plants within the three plant families have overall distinctive uses which concur in both regions. On the other hand, PCA by uses (Figure $5 \mathrm{~b}$ ) reveals that the gastrointestinal system is distinctive in the number of uses as compared to all the other systems, which are all clustered together. This goes in accordance with the study by GonzálezTejero and co-workers (2008), showing that in Albania, Egypt, Cyprus and Algeria, gastrointestinal ailments ranked 
in the first position, while in Spain and Morocco, these ranked in the second place. In south-western Sardinia and Arrabida Natural Park (Portugal), Maxia and co-workers (2008) and Novais and co-workers (2004), respectively reported that the highest number of herbal uses were for the gastrointestinal system.

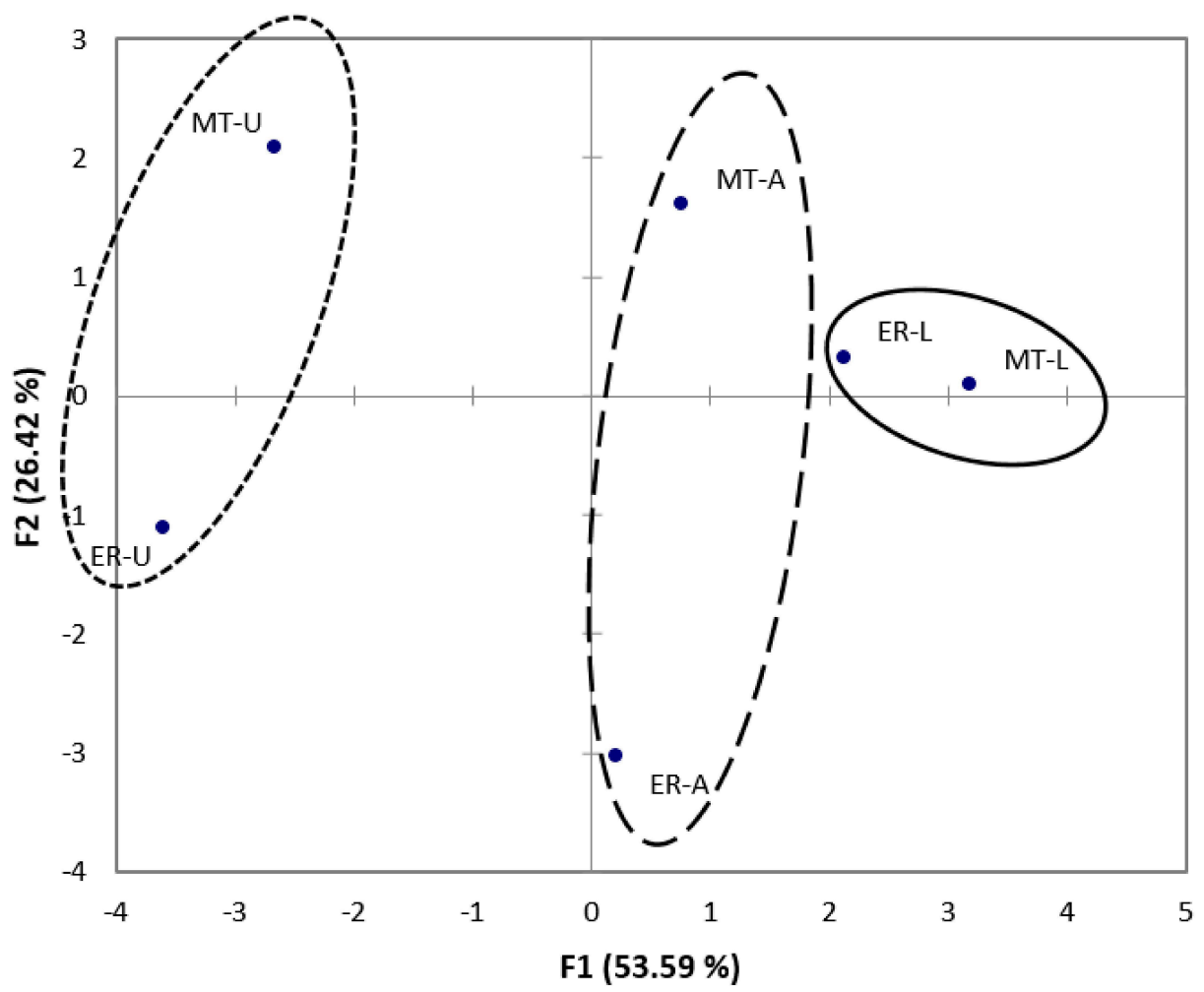

Figure 5a. Principal Component Analysis of the data - analysis of data by plant family and region

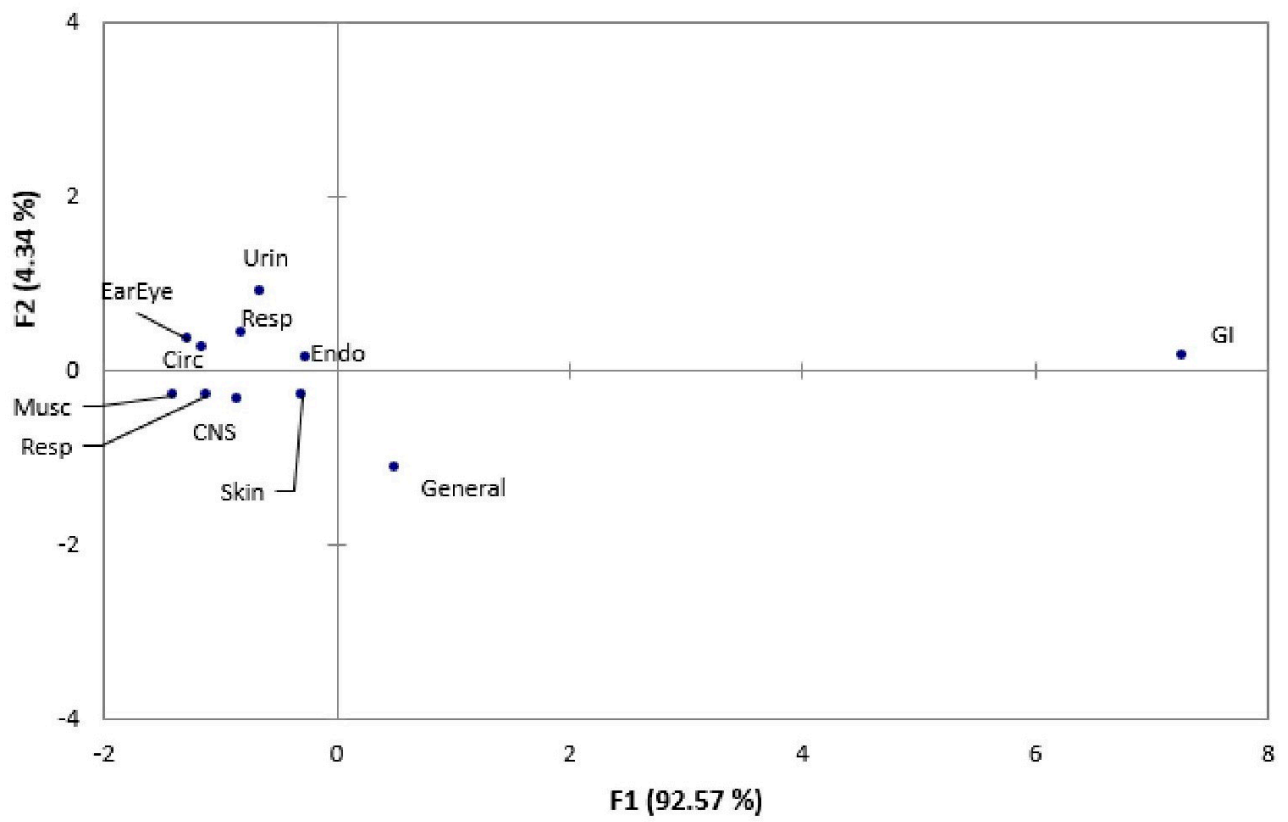

Figure 5b. Principal Component Analysis of the data - analysis of data by therapeutic use.

According to Bulut and co-workers (2017), gastrointestinal ailments ranked in the fourth position after shortness of breath, abdominal pain, and wound healing. The same was reported by Carrio and Valles (2012). It is well known scientifically that the overall health status of an individual depends on the composition of the diet and the microbiota of the gut (Rajoka et al. 2017). This shows that since antiquity, healers were aware of this relationship, hence the popularity of the use of herbal remedies for gastrointestinal problems. For Asteraceae species, the 
treatment of gastrointestinal disorders relates to the presence of sesquiterpene lactones (Heinrich et al. 1998) whereas in Lamiaceae and Apiaceae species, the calming effects are exhibited by terpenoids (Okach et al. 2013) which may be present in essential oils (Sayed-Ahmad et al. 2017).

\section{Conclusion}

This study was conducted to determine whether the same plants were used for medicinal purpose in the past for two regions within the Mediterranean region and whether the traditional use of medicinal plants concur within these two regions. These two regions have different topographies which may be perceived as a potential diversification of their flora. Despite this, it can be concluded from this study, that a number of medicinal plants particularly in three plant families, Asteraceae, Lamiaceae and Apiaceae, were used in both regions. Moreover, it was also revealed that the most targeted therapeutic system was the gastrointestinal system that covers several ailments mainly gastric disturbances (hyperacidity, ulceration, and indigestion), liver and biliary conditions and intestinal problems (spasms, worms and constipation). In the tradition of these regions, it was well understood that the maintenance of a healthy digestive system influences the overall health of an individual. Nowadays, scientific evidence proves this (Hollister et al. 2014) and in fact apart from medicinal products, a number pre- and probiotics are placed on the market to achieve this effect.

\section{Declarations}

List of abbreviations: CNS - Central Nervous System; ER - Emilia Romagnia; GI - Gastrointestinal; ISTAT - Istituto Nazionale di Statistica; MT - Malta; NSO - National Statistics Office (Malta); PCA - Principal Component Analysis

Ethics approval and consent to participate: The study was a meta-analysis of secondary information obtained from bibliographic sources. Published information was analyzed statistically for any possible similarities. No human or animal subjects were used in this study. The request was submitted to the University of Malta Research Ethics Committee and was given the following ID: 150620209488.

Consent for publication: Not applicable

Availability of data and materials: The information utilized in this study, is all found in the bibliography cited within this study.

Competing interests: There are no financial and non-financial competing interests to declare.

Funding: The study was not funded by any programme. The research resulted from a collaboration between two Universities. ERASMUS + programme served as a means of collaboration between the two Universities.

Authors' contributions: Flavia Galuzzo is the main author of the manuscript. She searched for the background information. Everaldo Attard is the Maltese supervisor for the student. He was involved in the interpretation and analysis of data obtained from Maltese sources. Diana di Gioia is the Italian supervisor for the student. She was involved in the interpretation and analysis of data obtained from Italian sources. All authors contributed equally in the writing of the manuscript.

\section{Acknowledgements}

This research was supported by a collaboration between the UM and UniBo through the ERASMUS+ program.

\section{References}

Agelet A, Vallès J. 2001. Studies of pharmaceutical ethnobotany in the regions of Pallars (Pyrenees, Catalonia, Iberian Peninsula). Part I. General results and new or very rare medicinal plants. Journal of Ethnopharmacology 77:57-70.

Akerreta S, Cavero RY, Calvo MI. 2007. First comprehensive contribution to medical ethnobotany of Western Pyrenees. Journal of Ethnobiology and Ethnomedicine 3:26.

Attard E, Cuschieri A. 2009. In vitro immunomodulatory activity of various extracts of Maltese plants from the Asteraceae family. Journal of Medicinal Plants Research 3:457-461.

Attard E, Pacioni P. 2012. The phytochemical and in vitro pharmacological testing of Maltese medicinal plants. In Bioactive Compounds in Phytomedicine. Edited by Rasooli I. InTech, Croatia, Pp. 93-112.

Attard E, Attard H, Tanti A, Azzopardi J, Sciberras M, Pace V, Buttigieg N, Randon Mangion A, Rossi B, Parnis MJ, Vella K, Zammit M, Serracino Inglott A. 2015. The Phytochemical Constitution of Maltese Medicinal PlantsPropagation, Isolation and Pharmacological Testing. In Phytochemicals: Isolation, Characterisation and Role in Human Health. Edited by Rao V, Rao L. InTech, Croatia. Pp. 3-44. 
Bhardwaj K, Bhushan B, Kumar R, Guleria S, Kumar H. 2019. Ethnomedicinal Remedy for Gastrointestinal Disorders in Rural and Remote areas of Jammu and Kashmir: A Review. Biological Forum-An International Journal 11:137148.

Bonet MÀ, Parada M, Selga A, Vallès J. 1999. Studies on pharmaceutical ethnobotany in the regions of L'Alt Empordá and Les Guilleries (Catalonia, Iberian Peninsula). Journal of Ethnopharmacology 68:145-168.

Bulut G, Haznedaroğlu MZ, Doğan A, Koyu H, Tuzlacı E. 2017. An ethnobotanical study of medicinal plants in Acipayam (Denizli-Turkey). Journal of Herbal Medicine 10:64-81.

Bulut G, Tuzlaci E. 2015. An ethnobotanical study of medicinal plants in Bayramic (Canakkale-Turkey). Marmara Pharmaceutical Journal 19:269-282.

Carrió E, Vallès J. 2012. Ethnobotany of medicinal plants used in eastern Mallorca (Balearic Islands, Mediterranean Sea). Journal of Ethnopharmacology 141:1021-1040.

Caruana U, Attard E. 2016. An ethno botanical survey of medicinal plants used in the Island of Gozo. Studies on Ethno-Medicine 10:269-281.

Di Castro F. 1981. Mediterranean-type shrublands of the world. In Mediterranean-type shrublands. Edited by Di Castro F, Goodall DW, Specht RL. Elsevier Scientific, Amsterdam, Pp. 1-52.

Emberger L. 1954. Projet d'une classification des climates, du point de vue phytogéographique. Bulletin de la Société d'histoire naturelle de Toulouse. 78:159-180.

González-Tejero MR, Casares-Porcel M, Sánchez-Rojas CP, Ramiro-Gutiérrez JM, Molero-Mesa J, Pieroni A, Giusti ME, Censorii E, de Pasquale C, Della A, Paraskeva-Hadijchambi D, Hadjichambis A, Houmani Z, El-Demerdash M, ElZayat M, Hmamouchi M, Eljohrig S. 2008. Medicinal plants in the Mediterranean area: synthesis of the results of the project Rubia. Journal of Ethnopharmacology 116:341-357.

Greuter W. 1991. Botanical diversity, endemism, rarity, and extinction in the Mediterranean area: an analysis based on the published volumes of Med-Checklist. Botanika Chronika 10:63-79.

Hadjichambis AC, Paraskeva-Hadjichambi D, Della A, Giusti ME, De Pasquale C, Lenzarini C, Censorii E, GonzálesTejero MR, Sanchez-Rojas CP, Ramiro-Gutierrez JM, Skoula M, Johnson C, Sarpaki A, Hmamouchi M, Jorhi S, ElDemerdash M, El-Zayat M, Pieroni A. 2008. Wild and semi-domesticated food plant consumption in seven circumMediterranean areas. International Journal of Food Sciences and Nutrition 59:383-414.

Heinrich M, Robles M, West JE, Ortiz de Montellano BR, Rodriguez E. 1998. Ethnopharmacology of Mexican asteraceae (compositae). Annual Review of Pharmacology and Toxicology 38:539-565.

Hollister EB, Gao C, Versalovic J. 2014. Compositional and functional features of the gastrointestinal microbiome and their effects on human health. Gastroenterology 146:1449-1458.

Johns T, Kokwaro JO, Kimanani EK. 1990. Herbal Remedies of the Luo of Siaya District, Kenya: Establishing Quantitative Criteria for Consensus. Economic Botany 44:369-381.

Laghetti G, Perrino P, Cifarelli S, Scicluna-Spiteri A, Attard E, Hammer K. 2004. Collecting crop genetic resources in the Mediterranean agricultural islands: Maltese archipelago. Plant Genetic Resources Newsletter. 139:1-16.

Lanfranco G. 2000. Hxejjex medičinali u ohrajn fil-Gżejjer Maltin. Pubblikazzjoni Tal-Media Centre, Blata l-Bajda, Malta.

Lanzara P. 1978. Guida alle piante medicinali. Arnoldo Mondadori Editore, Milan, Italy.

Lazzarini E. 1992. Le Piante Medicinali: Erbe Spontanee e coltivate dell'Emilia Romagna. Bologna, Italy.

Lazzarini E. 1996. Flora officinale di Romagna. A. Longo Editore. Ravenna, Italy.

Maxia A, Lancioni MC, Balia AN, Alborghetti R, Pieroni A, Loi MC. 2008. Medical ethnobotany of the Tabarkins, a Northern Italian (Ligurian) minority in south-western Sardinia. Genetic Resources and Crop Evolution 55:911-924.

Nawash O, Al-Assaf A, El-oqlah A, Omari M. 2014. Floristic features, distribution, and ethnobotany of plants gathered and used by local people from the Mediterranean forest in Northern Jordan. Ethnobotany Research and Applications 12:385-396.

Novais MH, Santos I, Mendes S, Pinto-Gomes C. 2004. Studies on pharmaceutical ethnobotany in Arrábida Natural Park (Portugal). Journal of Ethnopharmacology 93:183-195.

Okach DO, Nyunja ARO, Opande G. 2013. Phytochemical screening of some wild plants from Lamiaceae and their role in traditional medicine in Uriri District-Kenya. International Journal of Herbal Medicine 1:135-143.

Penza C. 1969. Il-flora Maltija medicinali. Progress Press, Mrieћel, Malta. 
Pieroni A, Cattero V. 2019. Wild vegetables do not lie: Comparative gastronomic ethnobotany and ethnolinguistics on the Greek traces of the Mediterranean Diet of southeastern Italy. Acta Botanica Brasilica 33:198-211.

Pignatti S. 1982. Flora d'Italia. Volume II, Edagricole, Bologna, Italy.

Rajoka MSR, Shi J, Mehwish HM, Zhu J, Li Q, Shao D, Huang Q, Yang H. 2017. Interaction between diet composition and gut microbiota and its impact on gastrointestinal tract health. Food Science and Human Wellness 6:121-130.

Rivas-Martínez S, Rivas-Sáenz S, Penas A. 2001. Worldwide bioclimatic classification system. Global Geobotany 1:1638.

Rivera D, Obon C, Inocencio C, Heinrich M, Verde A, Fajardo J, Llorach R. 2005. The ethnobotanical study of local Mediterranean food plants as medicinal resources in Southern Spain. Journal of Physiology and Pharmacology Supplement. 56:97-114.

Sánchez-Mata D, Morales R. 2016. The Mediterranean Landscape and Wild Edible Plants. In Mediterranean Wild Edible Plants. Edited by Sánchez-Mata MdC, Tardío J. Springer, New York. U.D.A. Pp. 15-31.

Savo V, Caneva G, Camarda I. 2010. Plants, history and cultures in the Mediterranean area.

Sayed-Ahmad B, Talou T, Saad Z, Hijazi A, Merah O. 2017. The Apiaceae: Ethnomedicinal family as source for industrial uses. Industrial Crops and Products 109:661-671. 